\title{
Prevalence and factors associated with episiotomy practice among primiparous women in mulago national referral hospital Uganda
}

\begin{abstract}
Background; Episiotomy, an obstetric procedure introduced into practice without any clear scientific evidence showing its benefits, became almost a procedure performed on all parturient women. Recently, a liberal episiotomy has been discouraged and WHO recommends an episiotomy rate of about $10 \%$ or less. The procedure substantially increases the risk of anal sphincter damage, improper wound healing, hematoma, infections, and perineal pain. The study was aimed at investigating the prevalence and factors associated with episiotomy among primiparous parturients in Mulago National Referral.
\end{abstract}

Methods: A cross-sectional study using a researcher administered questionnaires was conducted in Hospital Obstetrics and Gynecological Department in February and March 2018. Two hundred forty-nine participants were systematically recruited on the first postnatal day after meeting the inclusion criteria and the socio-demographic and obstetric characteristics were recorded. Logistic regression was used to determine the factors associated with the occurrences of episiotomy.

Results: The prevalence of episiotomy was 73\% (181/249) (CI 67-78). Mothers whose second stage of labor lasted between; 31-60 minutes were 3.6 times more likely to be made an episiotomy, $(\mathrm{CI} ; 1.66-7.86, \mathrm{p}=0.001)$, the odds further doubles if the second stage of labor was prolonged, lasting 60 minutes or greater $\mathrm{OR}=7.2(\mathrm{CI} ; 1.46-35.64, \mathrm{p}=0.015)$. Episiotomy was also found to be associated with gestational age above 37 weeks $\mathrm{OR}=1.8$ (CI; $1.28-2.40 \mathrm{p}<0.001)$.

Conclusion: The prevalence of episiotomy among primiparous is high yet higher episiotomy rates are associated with increasing morbidities and lack of benefits. The factors associated with episiotomy practice were gestational age above 37 weeks and prolonged second stage.
Volume 5 Issue 6 - 2019

\section{Pebalo Francis Pebolo, ' Ajeani Judith, ${ }^{2}$ Kaye Kabonge Dan'}

'Reproductive Heath, Gulu University, Uganda

${ }^{2}$ Obstetrics and Gynecology, Mulago National Referral Hospital, Uganda

${ }^{3}$ Obstetrics and Gynaecology, Makerere College of Health Sciences, Makerere University, Uganda

Correspondence: Francis Pebalo Pebolo, Department of Reproductive Health, Gulu University, P O Box 166, Gulu, Uganda,Tel +256772557775, Email pebalopebol@gmail.com

Received: August 08, 2019 | Published: November 01, 2019
Abbreviations: JHO, junior house officer ; MNRH, mulago national referral hospital; OASIS obstetric anal sphincter injuries ; SHO, senior house officer; WHO, world health organization ; REEDA, redness ecchymosis edema discharge and apposition; NPS, numerical pain scale.

\section{Introduction}

Episiotomy is one of the most widely practiced obstetric procedures and refers to a surgical incision on the perineum during the second stage of labor to enlarge the diameter of the vulval outlet to facilitate passage of the fetal head and prevent an uncontrolled tear of the perineal tissue. ${ }^{1-3}$ Episiotomy was introduced into obstetric practice without any scientific evidence corroborating any possible benefits and spread in the twentieth century. A full account of lacking scientific data about episiotomy and the potential danger such as hematoma formation, perineal pain, infections, sexual dysfunctions and healing complication associated with the procedure was brought to clarity in $1983 .{ }^{3}$ Recently some authors have questioned if there is any indication for episiotomy and whether if practiced selectively it can confer any benefit at all. ${ }^{4}$ Indications such as a prolonged second stage, macrosomia, non-reassuring fetal heart rate, instrumental delivery, occiput posterior position, and shoulder dystocia have been questioned. ${ }^{5}$ The incision substantially increases maternal blood loss, the average depth of posterior perineal injury, risk of anal sphincter damage, improper wound healing and increased amount of pain in the immediate postpartum period. ${ }^{6,7}$ Episiotomy at the first vaginal birth significantly and independently increases the risk of repeated episiotomy and spontaneous tears in subsequent delivery. ${ }^{8}$ Routine episiotomy is now considered to be a form of obstetric violence when a woman is automatically transformed into a patient and when routine medical procedures are carried out without giving the woman the right to make her own decisions concerning her own body. ${ }^{9}$ Cochrane reviews in developed countries have shown that restrictive episiotomy has several benefits like less severe posterior perineal trauma, less suturing, fewer healing complications and reduced risks of mother to child HIV transmission; however, it increases the risk of anterior perineal trauma. Although data about episiotomy and perineal tears are sparse in low-income countries, it remains one of the most commonly performed procedures in obstetrics. ${ }^{10} \mathrm{WHO}$ has recommended a restricted episiotomy rate of not more than $10 \%{ }^{11}$ and in Uganda, a study done in 2003 revealed that episiotomy rate among both primiparous and multiparous was $39.6 \% .{ }^{12}$ Primigravida is a 
known risk factor for episiotomy and some study had found that 59.9$90 \%$ of Primiparous undergo episiotomy. ${ }^{6,13}$ This study was aimed at assessing the prevalence of episiotomy and associated factors among primiparous women who delivered from Mulago National Referral Hospital.

\section{Material and methods}

\section{Study design}

This was a hospital-based cross-sectional study conducted at Mulago National Referral Hospital labor suit, post-natal wards and clinics in Kampala Uganda. Study participants were screened using eligibility criteria forms, Informed consent was sought and interviewer-administered questionnaires were used to collect explanatory variables such as the maternal age, gestation age (weeks), marital status, baby's weight, duration of second stage of labor, type of vaginal delivery (breech extractions, assisted vaginal births and spontaneous vaginal deliveries) and skilled attendants at delivery, between those who had episiotomy and those who had no episiotomy. Morbidity measured using REEDA (Redness, Ecchymosis, Edema, Discharge and Apposition) Scale and NPS (Numerical Pain Scale) were recorded at baseline and a follow up REEDA and NPS was recorded on day 14 postpartum. Two research assistants trained on REEDA and NPS measurements and research protocols collected the data using the questionnaire.

\section{Study procedures}

Participants were recruited on the first post-natal day from the post-natal ward after sample frame and unit had been drawn from the delivery book in labor suit. Participants were assigned numbers in ascending order and sample units arrived at by systematically selecting the alternate number of the participants. All primiparous mothers with an estimated gestational age of 28 weeks and above who had had vaginal delivery irrespective of the outcome were considered in the sampling frame. This study was approved by the School of Medicine Research and Ethics committee, Makerere University.

\section{Data management and analysis}

Each questionnaire was checked by the principal investigator for completeness at the end of data collection. Raw data were double entered into Epidata 3.1 and stored in a password-protected computer. Cleaned data were exported to Stata 13.0 for analysis. Descriptive statistics of the characteristic of the patients were presented as means or medians for continuous data and proportions [\%] for categorical variables. The prevalence of episiotomy procedure was presented as a proportion of primiparous who have had episiotomy to the total number of participants recruited. Demographic factors such as maternal age and obstetric factors such as duration of the second stage, gestational age were subjected to bivariate analysis and any factor with a p-value less or equal to 0.2 was subjected to multivariate logistic regression. Factors with a p-value less or equal to 0.05 were considered statistically significant. Adjusted odds ratios were calculated to quantify the strength of association.

\section{Results}

A total of 249 primiparous women who had vaginal deliveries in Mulago National Referral Hospital were recruited and included in the analysis. The participants were aged between 13 and 30 years, with a mean age of 20.9 years and a standard deviation of (SD) 2.9. The prevalence of episiotomy was found to be $73 \%$ (CI 67-78), with all episiotomy done being the mediolateral type. The majority of the study participants $213(85.5 \%)$ were married and had attained secondary school education $160(64.3 \%)$. More than half of the participants were housewives $131(52.6 \%)$ (Table 1).

Table I Demographic Characteristics of Study Participants

\begin{tabular}{lll}
\hline Characteristic & Frequency $(\mathbf{N}=\mathbf{2 4 9})$ & $\begin{array}{l}\text { Percentage } \\
\text { (\%) }\end{array}$ \\
\hline Marital status & 213 & 85.5 \\
Married & 32 & 12.9 \\
Single & 4 & 1.6 \\
Separated/Divorced & & \\
Education level & 8 & 3.2 \\
None & 61 & 24.5 \\
Primary & 160 & 64.3 \\
Secondary & 20 & 8 \\
College/University & & \\
Occupation & 131 & 52.6 \\
Housewife & 27 & 10.8 \\
Employed & 77 & 30.9 \\
Business & 14 & 5.6 \\
Others & & 85.9 \\
Religion & 214 & 14.1 \\
Christian & 35 & \\
Muslim & & \\
\hline
\end{tabular}

The mean gestational age (weeks) of pregnancy in this study was 38.9(+1.3) weeks. A big proportion of the women had the spontaneous onset of labor 196/ (78.7\%) and delivered by SVD 245 (99.2\%) (Table 2). The only episiotomy practiced in Mulago Hospital was mediolateral type, of which $3(1.7 \%)$ of the mothers had an extension of the episiotomy to a third-degree perineal tear. About half, $31(45.6 \%)$ of the study participants who had not received episiotomy had intact perineum. (54.4\%) sustained perineal tears, the majority of which $31(81.1 \%)$ were first degree and 7(18.9\%) were second-degree tears (Table 2).

Table 2 Obstetrics Characteristics of Study Participants

\begin{tabular}{lll}
\hline Characteristic & Frequency $(\mathbf{N}=\mathbf{2 4 9})$ & $\begin{array}{l}\text { Percentage } \\
(\%)\end{array}$ \\
\hline Marital status & 213 & 85.5 \\
Married & 32 & 12.9 \\
Single & 4 & 1.6 \\
Separated/Divorced & & \\
Education level & 8 & 3.2 \\
None & 61 & 24.5 \\
Primary & 160 & 64.3 \\
Secondary & 20 & 8 \\
College/University & & \\
Occupation & 131 & 52.6 \\
Housewife & 27 & 10.8 \\
Employed & 77 & 30.9 \\
Business & 14 & 5.6 \\
Others & & 85.9 \\
Religion & 214 & 14.1 \\
Christian & 35 & \\
Muslim & & \\
\hline
\end{tabular}


The majority of the deliveries $181(72.4 \%)$ were attended to by midwives and $125(69.1 \%)$ of them had episiotomy done. All deliveries conducted by SHO ( $\mathrm{n}=31) 100 \%$ had episiotomy done (Table 3). A higher percentage of the study participants who were in the second stage for more than $30-60$ minutes $(85 \%)$ or more than 60 minutes $(94.3 \%)$ have had episiotomy done (Table 4). The odd of getting episiotomy were 3.6 times (p-value 0.001 ) if the second stage of labor had lasted between 30-60minutes, this further increased to 7.2 times (p-value *0.015) if the second stage of labor had lasted longer than 60 minutes (Table 5).

Table 3 Skilled birth attendant and episiotomy rates

\begin{tabular}{llll}
\hline Characteristics & $\begin{array}{l}\text { No } \\
\text { episiotomy }\end{array}$ & Episiotomy & Total \\
\hline $\begin{array}{l}\text { Midwife trainee/Med } \\
\text { student }\end{array}$ & $\mathrm{II}(33.3 \%)$ & $22(66.7 \%)$ & $33(100 \%)$ \\
Midwife & $56(30.9 \%)$ & $\mathrm{I} 25(69.1 \%)$ & $\mathrm{I} 18 \mathrm{I}(100 \%)$ \\
Intern nurse & $\mathrm{I}(33.3 \%)$ & $2(66.7 \%)$ & $3(100 \%)$ \\
$\mathrm{JHO}$ & $0(0.0 \%)$ & $\mathrm{I}(100 \%)$ & $\mathrm{I}(100)$ \\
$\mathrm{SHO}$ & $0(0.0 \%)$ & $3 \mathrm{I}(100 \%)$ & $3 \mathrm{I}(100 \%)$ \\
Total & $68(27.3 \%)$ & $\mathrm{I} 8 \mathrm{I}(72.7 \%)$ & $249(100 \%)$ \\
\hline
\end{tabular}

SHO, Senior House Officer; JHO, Junior House Officer.

Table 4 Relationship Between Demographics/Obstetrics Factors and Episiotomy

\begin{tabular}{|c|c|c|}
\hline Category & $\begin{array}{l}\text { No } \\
\text { Episiotomy }\end{array}$ & Episiotomy \\
\hline \multicolumn{3}{|l|}{ Age } \\
\hline $18-22$ & $4 I(24.9)$ & $124(75.1)$ \\
\hline$<18$ & $6(42.9)$ & $8(57.1)$ \\
\hline$>22$ & $2 \mathrm{I}(30.4)$ & $48(69.6)$ \\
\hline \multicolumn{3}{|l|}{ Religion } \\
\hline Christian & $55(25.7)$ & $159(74.3)$ \\
\hline Muslim & $13(37.1)$ & $22(68.9)$ \\
\hline \multicolumn{3}{|c|}{ Gestational Age(weeks) } \\
\hline$<37$ & $6(85.7)$ & $\mathrm{I}(14.3)$ \\
\hline$\geq 37$ & $59(27.7)$ & $154(72.3)$ \\
\hline \multicolumn{3}{|c|}{ Duration of the second stage } \\
\hline$<30$ & $50(44.6)$ & $62(55.4)$ \\
\hline $31-60$ & $15(\mid 5.0)$ & $85(85.0)$ \\
\hline$>60$ & $2(5.7)$ & $33(94.3)$ \\
\hline \multicolumn{3}{|l|}{ Type of delivery } \\
\hline Breech/Vacuum & $\mathrm{I}(20.0)$ & $4(80.0)$ \\
\hline SVD & $67(27.4)$ & I78(72.6) \\
\hline \multicolumn{3}{|l|}{ Genital Cutting } \\
\hline No & $67(27.1)$ & $180(72.9)$ \\
\hline Yes & $\mathrm{I}(33.3)$ & $2(66.7)$ \\
\hline \multicolumn{3}{|c|}{ Apgar score at one minute } \\
\hline$>7$ & $57(25.1)$ & $170(74.9)$ \\
\hline
\end{tabular}

Table continued

\begin{tabular}{lcl} 
Category & $\begin{array}{l}\text { No } \\
\text { Episiotomy }\end{array}$ & Episiotomy \\
\hline$<7$ & $\mathrm{II}(52.4)$ & $10(47.6)$ \\
$\begin{array}{l}\text { Birth weight } \\
2.5-3.5\end{array}$ & \\
$>3.5$ & $57(28.5)$ & $143(71.5)$ \\
$<2.5$ & $5(13.9)$ & $31(86.1)$ \\
Who conducted delivery? & $6(50.0)$ \\
Other & $6(50.0)$ & \\
Midwife & I2(I7.4) & $57(82.6)$ \\
\hline
\end{tabular}

\section{Discussion}

\section{Prevalence of episiotomy}

A total of 249 participants were included in the analysis in this study, the prevalence of episiotomy was $73 \%$ (CI 66.9-78). This is higher than the rate in a similar cross-sectional study at Mulago by Oda $(39.6 \%){ }^{12}$ The difference was due to the characteristics of the study population. Our study recruited only prime-para while Oda et al study recruited both primiparous and women of higher parity. The high prevalence may also be due to the characteristics of the women who seek services at Mulago National Referral Hospital. These are women who are at high risk and most often referred to with complications. However, primiparity has been seen in many studies as an important risk factor for episiotomy and there had been varied data on the rate of episiotomy among primiparous women, rates ranging from as low as $33 \%$ to as high as $86 \%$ and $59-90 \%{ }^{6,13-16}$ has been reported. Similar surveys done in the Latin American hospitals reveal higher trends of episiotomy between $69.2 \%$ and $96.2 \%$ among primiparous women. ${ }^{17-21}$ Barnabas et al., ${ }^{22}$ in their retrospective study in Nigeria found out that the absence of prior vaginal birth and nulliparity is significantly associated with episiotomy

\section{Factors associated with the occurrence of episiotomy}

The factors that were found to be significantly associated with the occurrence of episiotomy in this study were the duration of the second stage and the gestation age of 37 or more. Participants whose pregnancy was 37 weeks or more were twice more likely to have an episiotomy compared to those less than 37 weeks in agreement with findings in a retrospective cohort study of all singleton vaginal deliveries at term between 2007 and 2014 done by Shmueli et al., ${ }^{18}$ in Ramat-Aviv, Israel, which showed that episiotomy is associated with increasing gestational age. ${ }^{18}$ Increase in gestation age is related to increasing size of the baby that may cause delayed second stage or increased risk of perineal tears as judged by the skilled birth attendants, this is paradoxical to the findings relating to the birth weights more than $3.5 \mathrm{~kg}$ that was seen to be not associated with the procedure (p-value 0.211).

A prime-para was four times more likely to have an episiotomy if the second stage of labor lasted between 31-60 minutes. The odds further doubled if the second stage of labor lasted for more than 60 minutes $(\mathrm{OR}=7.2, \mathrm{p}=0.015)$ compared to when it lasted less than 30 minutes as shown in table 5. This could be because of the fear of fetal compromise that normally results from the delayed second stage of labor hence the need to expedite the delivery process by performing an episiotomy. In Ugandan setting where electronic fetal monitoring is not readily available and uncommonly practiced, especially in a busy 
center like Mulago Hospital, where partograph monitoring maybe a little difficult, it is therefore certain that delayed second stage of labor for more than 30 minutes might prompt immediate delivery hence the need for an episiotomy. This finding agrees with other authors elsewhere where delay in the second stage of labor has been seen as a risk factor for an episiotomy. A prolonged expulsive phase of the second stage of labor has been seen as an important risk factor for episiotomy as reported by Giordana Campos BraGa et al 2014, in a case-control study in Brazil to assess the risk of episiotomy. ${ }^{19}$

There was no association between APGAR $<7$ supporting previous studies that found that episiotomy practice has failed to accomplish neonatal indications ascribed to it; hence the need for selective episiotomy practice as seen in a randomized control trial on selective episiotomy vs implementation of the non-episiotomy protocol in Brazil. ${ }^{5}$ Assisted vaginal and breech delivery are known risk factors associated with episiotomy, in a metanalysis by Graham et al. ${ }^{10}$ crosssectional study by Mohamed et al 2014 and in a retrospective study by Owa et al 2015 in Nigeria, ${ }^{10,16,20}$ the few numbers for assisted vaginal birth and breech delivery seen in this study might have not given enough power to make and observable relationship.

Table 5 Factors associated with the occurrence of episiotomy

\begin{tabular}{|c|c|c|c|c|c|c|}
\hline Category & cOR & $95 \% \mathrm{Cl}$ & p-value & AOR & $95 \% \mathrm{Cl}$ & p-value \\
\hline \multicolumn{7}{|l|}{ Maternal Age } \\
\hline$<18$ & 0.4 & $0.144-1.346$ & 0.15 & 2 & $0.429-9.372$ & 0.377 \\
\hline$>22$ & 8 & $0.406-1.409$ & 0.378 & 1.1 & $0.499-2.419$ & 0.81 \\
\hline $18-22$ & 1 & & & & & \\
\hline \multicolumn{7}{|l|}{ Onset Of Labour } \\
\hline Induction & 1 & & & & & \\
\hline Spontaneous & 3.1 & 1.629-5.776 & 0.001 & 1.7 & $0.810-3.719$ & 0.156 \\
\hline \multicolumn{7}{|l|}{ Gestational Age } \\
\hline$<37$ & & & 1 & 1 & & \\
\hline$\geq 37$ & 2 & $1.546-2.668$ & 0.001 & 1.8 & $1.281-2.399$ & $*<0.001$ \\
\hline \multicolumn{7}{|c|}{ Duration of second stage(Minutes) } \\
\hline$<30$ & & & 1 & & & \\
\hline $31-60$ & 4.6 & $2.354-8.872$ & $<0.001$ & 3.6 & $1.656-7.860$ & $* 0.001$ \\
\hline$>60$ & 13.3 & $3.044-58.169$ & 0.001 & 7.2 & I.458-35.635 & $* 0.015$ \\
\hline \multicolumn{7}{|c|}{ Type Of Vaginal Delivery } \\
\hline Breech/Vacuum & I & & & & & \\
\hline SVD & 0.7 & $0.073-6.050$ & 0.717 & 0.6 & $0.013-2.176$ & 0.172 \\
\hline \multicolumn{7}{|l|}{ Birth weight } \\
\hline$<2.5$ & 0.4 & $0.123-1.287$ & 0.124 & 0.3 & $0.056-1.876$ & 0.209 \\
\hline $2.5-3.5$ & & & 1 & & & \\
\hline$>3.5$ & 2.5 & $0.915-6.672$ & 0.074 & 2.1 & $0.655-6.802$ & 0.211 \\
\hline \multicolumn{7}{|c|}{ Apgar score at one minute } \\
\hline$>7$ & 1 & & & & & \\
\hline$<7$ & 0.3 & $0.123-0.755$ & 0.01 & 0.6 & $0.213-1.972$ & 0.445 \\
\hline \multicolumn{7}{|c|}{ Skilled Delivery Attendants } \\
\hline Others & I & & & 1 & & \\
\hline Midwife & 0.5 & $0.234-0.944$ & 0.034 & 0.9 & $0.349-2.098$ & 0.734 \\
\hline
\end{tabular}

*Significant. 
181 of the $249(72.7 \%)$ were attended to by the midwives of which $125(69.1 \%)$ has had episiotomies similar to the rate of episiotomies among supervised deliveries attended to by student trainees $(67.6 \%)$ as shown in table 3 . This reflects that most of the decision for episiotomy done by the trainees could have been made by the midwives who in the Mulago settings are the one supervising the trainees, supporting earlier findings in one study in Nigeria in which episiotomy was sometimes done to give students opportunities to learn. ${ }^{22}$ In contrast, all the deliveries ${ }^{31}$ conducted by the SHO have had episiotomy done. Although the association amongst the different delivery attendants was found to be statistically non-significant at multivariate analysis because of the low numbers conducted by other cadres, from the proportion it is clear that delivery conducted by doctors (SHO) is more associated with episiotomy procedures. In Mulago hospital where many high-risk mothers are being referred in, residents being the front line doctors do manage a significant number of complicated deliveries hence the increased proportion of episiotomy made by the SHO. This study agrees with findings from other studies that claimed being delivered by a doctor increases the risk of an episiotomy compared to those delivered by midwives ${ }^{21}$ and also findings by Owa et al 2017 who argued that most deliveries conducted by doctors are either instrumental or complicated hence the need to widen the introitus with an episiotomy to help expedite the delivery process. ${ }^{20}$

There was however no association between maternal age, and induction of labor with episiotomy in this study, although a study done in Israel showed that maternal age above 22 years was found to be protective for episiotomy and induction of labor was a risk factor for episiotomy procedu Confounding factors may not be re. ${ }^{18}$ equally distributed among those mothers who had episiotomy and those who had no episiotomy. Error in medical records, and recall bias from the patient may also be a limitation in the study.

\section{Conclusion}

The Prevalence rate of mediolateral episiotomy in this study was $73 \%$ of which 3 patients had an episiotomy extension to at least a third-degree perineal tears. Factors found to be significantly associated with Episiotomy were gestational age greater than 37 weeks, and the duration of the second stage of labor greater than 30 minutes.

\section{Acknowledgements}

None.

\section{Conflicts of interest}

The author declares that there is no conflict of interest.

\section{Funding details}

None.

\section{References}

1. Usha Dssadv. AClinical Study of Agaruvadi Yoni Dhupana on Episiotomy Wound. European Journal Of Biomedical and Pharmaceutical Sciences. 2016;3(3):322-327.

2. Borruto F CC. Episiotomy: A Too Often Unnecessary And Harmful Practice. MOJ Women's Health. 2016;2(1):00020.

3. Thacker SB, Banta HD. Benefits And Risks Of Episiotomy: An
Interpretative Review Of The English Language Literature, 1860-1980. Obstet Gynecol Surv. 1983;38(6):322-338.

4. Amorim M, Coutinho IC, Melo I, et al. Selective Episiotomy Vs. Implementation Of A Non-Episiotomy Protocol: A Randomized Clinical Trial. Reprod Health. 2017;14(1):55.

5. Amorim MM F-NA, Leal NV, Melo FO, et al. Is It Possible To Never Perform Episiotomy During Vaginal Delivery? Obstet Gynecol. 2014;123(Suppl 1):38S.

6. Chaithra. Episiotomy in Primigravida Routine Versus Selective At Chevalumba Hospital Mysore. Mmed Dissertation. 2012.

7. El-Nagger Mha. Effect Of Self Perineal Care Instructions On Episiotomy Pain And Wound Healing Of Postpartum Women. Journal Of American Science. 2012;8(6):640-6550.

8. D'Gregorio P. Obstetric Violence: A New Legal Term Introduced In Venezuela. Int J Gynaecol Obstet. 2010;111:201-202.

9. Graham Ian D, Carroli G, Davies C, et al. Episiotomy Rates Around The World: An Update. Birth. 2005;32(3):219-223.

10. WHO. WHO Recommendations For Prevention And Treatment Of Maternal Peripartum Infections. 2015.

11. Odar E, Wandabwa J, Kiondo P. Sexual Practices Of Women Within Six Months Of Childbirth In Mulago Hospital. Uganda Afr Health Sci. 2003;3(3):117-123.

12. Chigbu B OS, Alukaa Kamanu C, Adibe E. Factors Influencing The Use Of Episiotomy In Nigeria, Should Their Use Be Restricted? Niger Post Grad Med J. 2008;1(9):13-16.

13. Sagi-Dain SS. Morbidity Associated With Episiotomy In Vacuum Delivery: A Systematic Review And Meta-Analysis. BJOG. 2015;122:1073-1081.

14. Amaka N. Ocheke ES, Isaac E, et al. An Audit Of Perineal Trauma And Vertical Transmission Of HIV African Journal Of Reproductive Health. 2017;21(4):67.

15. Mohamed ELB, Younes A, Abderrazzak K, et al. Risk Factors Associated With The Practice Of Episiotomy And Perineal Tears Occurred In Mothers At The Maternity Hospital Sharif Idrissi In The Region Of Gharb Chrarda Bni Hssen (Morocco). International Journal Of Innovation And Applied Studies. 2014;7(3):911-919.

16. Althabe FB, José M Bergel, Eduardo. Episiotomy Rates In Primiparous Women In Latin America: Hospital-Based Descriptive Study. BMJ. 2002;324(7343):945-946.

17. Shmueli A, Gabbay Benziv R, Hiersch L, et al. Episiotomy - Risk Factors And Outcomes. The Journal Of Maternal-Fetal \& Neonatal Medicine. 2017;30(3):251-256.

18. Giordana Campos Braga, Patrícia Ferreira Neves Da Luz, Adriana Scavuzzi, et al. Risk Factors For Episiotomy: A Case-Control Study. Rev Assoc Med Bras. 2014;60(5):465-472.

19. Owa OO, Eniowo AR, Ilesanmi OS. Factors Associated With Episiotomy Among Parturients. Delivering In A Tertiary Care Centre In Nigeria. 2017;3(4):5

20. Hueston WJ. Factors Associated With The Use Of Episiotomy During Vaginal Delivery. Obstetrics \& Gynecology. 1996;87(6):1001-1005.

21. Barnabas T AI, Olusyo AAmale D A. Relative Frequency And Predictors Of Episiotomy In Ogbomoso Nigeria. Internat Journal Of Medical Update.: 2012;7(2):41-44 